\title{
EFICIENCIA EN LOS PROCESOS LOGÍSTICOS EN LAS EMPRESAS CERTIFICADAS EN BASC MEDELLÍN MEDIANTE ANÁLISIS ENVOLVENTE DE DATOS
}

\section{EFFICIENCY IN LOGISTICS PROCESSES IN MEDELLIN BASC CERTIFIED COMPANIES THROUGH DATA ENVELOPMENT ANALYSIS}

\author{
Tomás José Fontalvo Herrera ${ }^{1}$, Adel Mendoza Mendoza ${ }^{2}$, Delimiro Visbal Cadavid ${ }^{3}$
}

\begin{abstract}
${ }^{1}$ Doctor en Administración, Magíster en Administración de Empresas. Jefe de Departamento de Organización Industrial de la Facultad de Ciencias Económicas de la Universidad de Cartagena. Colombia, e-mail: tfontalvoh@unicartagena.edu.co; ${ }^{2}$ Magíster en Ingeniería Industrial, Docente de Planta de la Universidad del Atlántico, Programa de Ingeniería Industrial, e-mail: adelmendoza@mail.uniatlantico.edu.co; ${ }^{3}$ Magíster en Ingeniería Industrial, Docente de Planta de la Universidad del Magdalena, Programa de Ingeniería Industrial. Director de la Especialización en Logística, e-mail: dvisbal@unimagdalena.edu.co
\end{abstract}

Rev. U.D.C.A Act. \& Div. Cient. 17(1): 265-274, Enero-Junio, 2014

\section{RESUMEN}

El objetivo de esta investigación es estimar la eficiencia técnica de las empresas certificadas en BASC, ubicadas en Medellín, durante el 2010, mediante la metodología no paramétrica de Análisis Envolvente de Datos (DEA), utilizando el modelo CCR, orientado a salidas. Como fuente de información, se utilizaron los datos de la Superintendencia de Sociedades; se trabajó como variables de entrada: Subtotal de Inventario, Total Activo Corriente, Propiedad Planta y Equipo y Proveedores y, como variable de salida, los Ingresos Operacionales. De las empresas analizadas en el presente estudio, se observó que 7 de las 60 empresas certificadas en BASC presenta una eficiencia de $100 \%$. La eficiencia promedio del grupo empresarial estudiado fue de 31,85\%.

Palabras clave: Eficiencia Técnica, Análisis Envolvente de Datos, Modelo CCR.

\section{SUMMARY}

The aim of this research is to estimate the technical efficiency of firms certified in BASC located in Medellin in 2010, using the methodology no parametric Data Envelopment Analysis (DEA) using the output-oriented CCR model. As a source of information we used data from the Superintendence of Companies, is worked as input variables: Subtotal of inventory, Total Current Assets Property, Plant and Equipment and Suppliers, and as output variable: Operating Income. Of the companies analyzed in this study found that 7 of the 60 companies certified in BASC have an efficiency of $100 \%$. The average efficiency of the business group studied was $31.85 \%$

Key words: Technical Efficiency, Data Envelopment Analysis, CCR model.

\section{INTRODUCCIÓN}

La metodología del Análisis Envolvente de Datos fue desarrollada por Charnes, Cooper y Rhodes, con base en los conceptos planteados por Farrel, en 1957 (Charnes et al. 1978), la cual, es una técnica que utiliza la programación lineal, con el propósito de comparar unidades de producción que emplean el mismo grupo de recursos para producir el mismo grupo de productos, creando una frontera eficiente e indicadores relativos de eficiencia, dentro de la población de unidades de producción bajo estudios. El Análisis Envolvente de Datos (DEA) tiene como objetivo encontrar las unidades de producción, denominadas DMU (Decision Making Unit), que producen los niveles más altos de Outputs, mediante el uso de los niveles más bajos de Inputs. Por lo cual, maximiza la relación de los Outputs ponderados y los Inputs ponderadas para cada DMU en consideración, además, la maximización de este objetivo está sujeta a la restricción de que la misma proporción para todas las DMU debe ser menor o igual a 1 . Una DMU alcanza una eficiencia del $100 \%$, sólo si ninguna de sus entradas o salidas se pueden mejorar sin empeorar algunas de sus otras entradas o salidas. La metodología DEA permite desarrollar procesos de medición en organizaciones y procesos, pues posibilita el manejo de diversas entradas y 
salidas (Wong \& Wong, 2007). Los modelos básicos del Análisis Envolvente de Datos son el CCR (Charnes et al. 1978) y el BCC (Banker et al. 1984).

\section{El Modelo DEA-CCR.}

Si $Y_{o}=\left(y_{10}, y_{20}, y_{30}, \ldots, y_{s 0}\right)$ y $X_{o}=\left(x_{10}, x_{20}, x_{30}, \ldots, x_{m o}\right)$ representa, respectivamente, las cantidades de salidas (productos) y entradas (recursos) de la DMU0, la unidad productiva que está siendo evaluada, la medida escalar de la eficiencia de la DMUO puede ser obtenida como la solución óptima del siguiente modelo conceptual.

$$
\operatorname{Max}\left[=\frac{\sum_{\mathrm{r}=1}^{\mathrm{s}} \mathrm{u}_{\mathrm{ro}} \mathrm{y}_{\mathrm{ro}}}{\sum_{\mathrm{i}=1}^{\mathrm{m}} \mathrm{v}_{\mathrm{io}} \mathrm{x}_{\mathrm{io}}}\right.
$$

Sujeto a

$$
\begin{aligned}
& \frac{\sum_{\mathrm{r}=1}^{\mathrm{s}} \mathrm{u}_{\mathrm{rj}} \mathrm{y}_{\mathrm{rj}}}{\sum_{\mathrm{i}=1}^{\mathrm{m}} \mathrm{v}_{\mathrm{ij}} \mathrm{x}_{\mathrm{ij}}} \leq 1 \quad \mathrm{j}=1,2, \ldots \mathrm{n} \\
& u_{r j}, v_{i j} \geq 0 \quad r=1, \ldots s \quad i=1, \ldots . m
\end{aligned}
$$

En donde, $u_{r o} \mathrm{y} \mathrm{v}_{\mathrm{io}}$ es el conjunto de los pesos más favorables para la DMU 0 en el sentido de maximizar la razón anterior. Se asume que los datos son no negativos y que cada DMU tiene, al menos, un valor positivo, tanto en las entradas como en las salidas.

El modelo BCC. El modelo BCC difiere del modelo CCR, sólo por la adición de la restricción $\sum_{j=1}^{n} \lambda_{j}=1$, la cual, junto con la restricción $\lambda \geq 0$, para toda j, imponen una condición de convexidad al conjunto de producción posible.

La técnica Análisis Envolvente de Datos permite calcular el desempeño de la eficiencia de múltiples unidades productivas (Nijkamp et al. 2009). Dentro de las aplicaciones realizadas con esta técnica, se puede referenciar la evaluación de eficiencia en bancos (Primorac \& Troskot, 2005; Pastor et al. 2006; Pasiouras, 2008; Tortosa-Ausina et al. 2008), en la evaluación de riesgos crediticios (Paradi et al. 2004; Psillaki et al. 2010), en la evaluación de alternativas de diseño de instalaciones (Toloo \& Nalchigar, 2009) y en la asignación de recursos (Hadi-Vencheh et al. 2008).

En la aplicación de la técnica DEA, se requiere que las unidades productivas, objeto de estudio seleccionadas, cumplan las siguientes condiciones: ser homogéneas para que se puedan comparar y heterogéneas para captar datos e información que haga posible la constatación de las unidades productivas; deben gestionar recursos asociados a las variables de entrada y de salida y ser suficientes para no limitar la capacidad discriminatoria del análisis (Pino et al. 2010).
Coll \& Blasco (2007) utilizan la técnica y afirman que el modelo DEA-CCR tiene como intencionalidad optimizar los pesos, que permitan maximizar la eficiencia relativa de una unidad productiva evaluada. Perdomo \& Mendieta (2007), dentro de sus estudios utilizan el Análisis Envolvente de Datos para analizar la relación del nivel producido, el uso óptimo de los recursos utilizados para el desarrollo de la producción y los precios acordes con lo anterior. Otros autores evaluaron la utilidad del Análisis Envolvente de Datos como método para la determinación de objetivos de gestión con respecto a los insumos requeridos y los resultados alcanzados (García \& González, 2005).

Quintanilha \& Correia (2012) utilizaron la metodología en un grupo de empresas de aviación en Brasil y calcularon su eficiencia comparando sus resultados y desempeños con el modelo de Análisis Envolvente de Datos (DEA). Esta metodología permite analizar cómo los procesos productivos pueden tener mejores resultados cuando se utilizan iguales recursos; lo anterior, teniendo en cuenta que las organizaciones deben mejor su productividad y su competitividad para generar preferencia y posicionarse en el contexto donde ofrecen sus productos y servicios (Belmonte \& Plaza, 2008).

El cálculo de la eficiencia es importante para la toma de decisiones así como para asignar responsabilidades, pues facilita determinar las intencionalidades de la organización y los insumos requeridos para lograrla (Martín et al. 2007). Para poder alcanzar la competitividad, las organizaciones no solamente tienen que poder producir y entregar productos y servicios de forma segura y oportuna, sino deben optimizar los procesos logísticos que garanticen lo anterior. En este sentido, se puede lograr mejores niveles de eficiencia y productividad, que le permitan a las organizaciones competir y lograr la sostenibilidad en el mercado a mediano y largo plazo. Para conseguir lo anterior, es necesario que las empresas utilicen criterios de racionalidad económica, que permita que los procesos de producción alcancen mejores niveles de eficiencia, estudiando, para éstos, la relación de las variables de entrada y de salida y su impacto en la las empresas.

Por otro lado, las condiciones de la economía mundial actual y el libre comercio entre grupos económicos y países, implica una nueva concepción de los procesos logísticos para lograr las importaciones y las exportaciones entre estos grupos y países, para lo cual, es necesario mejorar los niveles de seguridad, productividad y eficiencia de las empresas. Estas condiciones económicas requieren el posicionamiento y la ampliación de sus mercados para sostenerse en los contextos donde intervienen. Lo anterior, requiere la optimización de los costos productivos, que permitan mejorar los márgenes de contribución y, de esta forma, se refleje en los estados de resultados de las compañías. 
Los Tratados de Libre Comercio (TLC) implican un conjunto de retos para la implementación de los procesos logísticos, que garantice la entrega de productos o servicios de forma segura (Mejía \& Castro, 2007). Por lo anterior, se requiere el diseño e implementación de normas, como el estándar BASC, que contribuya el mejoramiento de los procesos, su legalidad y la seguridad al interior de las organizaciones y, de esta forma, optimizar la cadena de suministro en las operaciones comerciales entre países. Por estas nuevas condiciones que la internacionalización de la economía requiere, la Coalición Empresarial Anti Contrabando (BASC, por sus siglas en inglés), nace para dar respuestas a temas de gestión de control y seguridad. Según Hernández (2004), el diseño, la implementación y la certificación de este estándar logístico internacional facilita la entregar de productos de forma legal, oportuna, segura y garantiza las transacciones comerciales de forma ágil.

Considerando los planteamientos presentados, en este trabajo de investigación se da respuesta a los siguientes interrogantes: ¿Cómo calcular la eficiencia de las empresas de Medellín certificadas con BASC?, ċuál de las empresas certificadas en BASC, en la ciudad de Medellín, tienen mejor desempeño en su eficiencia?, ¿cómo incide el proceso de certificación de las empresas de Medellín certificadas el 2010 en BASC en el mejoramiento de los niveles de eficiencia de éstas?

\section{MATERIALES Y MÉTODOS}

Esta es una investigación en donde se utiliza un análisis cualitativo, descriptivo, propositivo y cuantitativo, soportada en el Análisis Envolvente de Datos (DEA), mediante el modelo CCR orientado a salidas (CCR - O). Se utiliza el modelo CCR y no el modelo BCC, ya que mediante el primero, una empresa puede ser comparada con otras sustancialmente más grandes o más pequeñas, mientras que con el modelo BCC una empresa es comparada con otras lo más similares posibles a su tamaño (Quindos et al. 2003).

Como fuente de información, se utilizaron los estados financieros disponibles en la Superintendencia de Sociedades del 2010, de donde se tomaron los diferentes rubros financieros de las organizaciones, consideradas en el estudio. Inicialmente, se realizó la selección de variables teniendo en cuenta que con estas variables se pueda obtener una buena discriminación entre las unidades eficientes e ineficientes, de acuerdo a la correlación que exista entre las variables seleccionadas. De los estados financieros de las empresas estudiadas, se seleccionaron los indicadores para aplicar la metodología. En particular, se trabajó como variables de entrada Subtotal Inventarios, Activo Corriente, Propiedades Planta y Equipos y Proveedores; como variables de salida, los Ingresos Operacionales. Los valores de estas variables se muestran en la tabla 1.

Tabla 1. Magnitud de las variables de entradas y salidas de las empresas certificadas en BASC Medellín, 2010.

\begin{tabular}{|l|c|c|c|c|c|}
\hline \multicolumn{1}{|c|}{ Empresa } & $\begin{array}{c}\text { (I) Subtotal } \\
\text { Inventarios }\end{array}$ & $\begin{array}{c}\text { (I) Total Activo Co- } \\
\text { rriente }\end{array}$ & $\begin{array}{c}\text { (I) Propiedades } \\
\text { Planta y Equipo }\end{array}$ & (I) Proveedores & $\begin{array}{c}\text { (O) Ingresos } \\
\text { Operacionales }\end{array}$ \\
\hline Empresa 1 & 88.839 .865 & 200.743 .506 & 59.732 .978 & 59.473 .205 & 341.709 .905 \\
\hline Empresa 2 & 1.394 .487 & 8.597 .527 & 1.925 .104 & 1.869 .386 & 18.266 .276 \\
\hline Empresa 3 & 4.998 .004 & 25.786 .531 & 610.259 & 5.652 .512 & 46.578 .961 \\
\hline Empresa 4 & 11.975 .188 & 29.260 .702 & 36.232 .085 & 421.711 & 91.810 .613 \\
\hline Empresa 5 & 0 & 480.635 & 9.692 & 0 & 118.685 \\
\hline Empresa 6 & 15.643 .123 & 22.443 .190 & 15.449 .671 & 15.737 .640 & 88.441 .048 \\
\hline Empresa 7 & 265.601 & 2.183 .782 & 428.609 & 699.446 & 10.611 .064 \\
\hline Empresa 8 & 6.019 .889 & 14.730 .889 & 15.907 .911 & 1.598 .589 & 39.482 .714 \\
\hline Empresa 9 & 0 & 875.831 & 3.251 .208 & 0 & 5.367 .191 \\
\hline Empresa 10 & 6.587 .127 & 32.748 .006 & 2.831 .031 & 2.930 .564 & 24.675 .571 \\
\hline Empresa 11 & 281.684 & 2.655 .524 & 989.679 & 577.585 & 7.549 .278 \\
\hline Empresa 12 & 1.571 .837 & 3.474 .093 & 826.253 & 10.618 & 3.032 .529 \\
\hline Empresa 13 & 9.697 .962 & 36.651 .101 & 3.400 .022 & 15.976 .341 & 122.225 .414 \\
\hline Empresa 14 & 2.705 .445 & 8.711 .090 & 3.575 .333 & 3.604 .788 & 21.609 .208 \\
\hline Empresa 15 & 0 & 578.772 & 439.066 & 38.581 & 1.987 .395 \\
\hline Empresa 16 & 2.172 .759 & 4.425 .079 & 1.650 .190 & 101.646 & 4.974 .358 \\
\hline Empresa 17 & 634.957 & 10.242 .237 & 7.429 .097 & 227.736 & 16.013 .598 \\
\hline
\end{tabular}


Continuación Tabla 1.

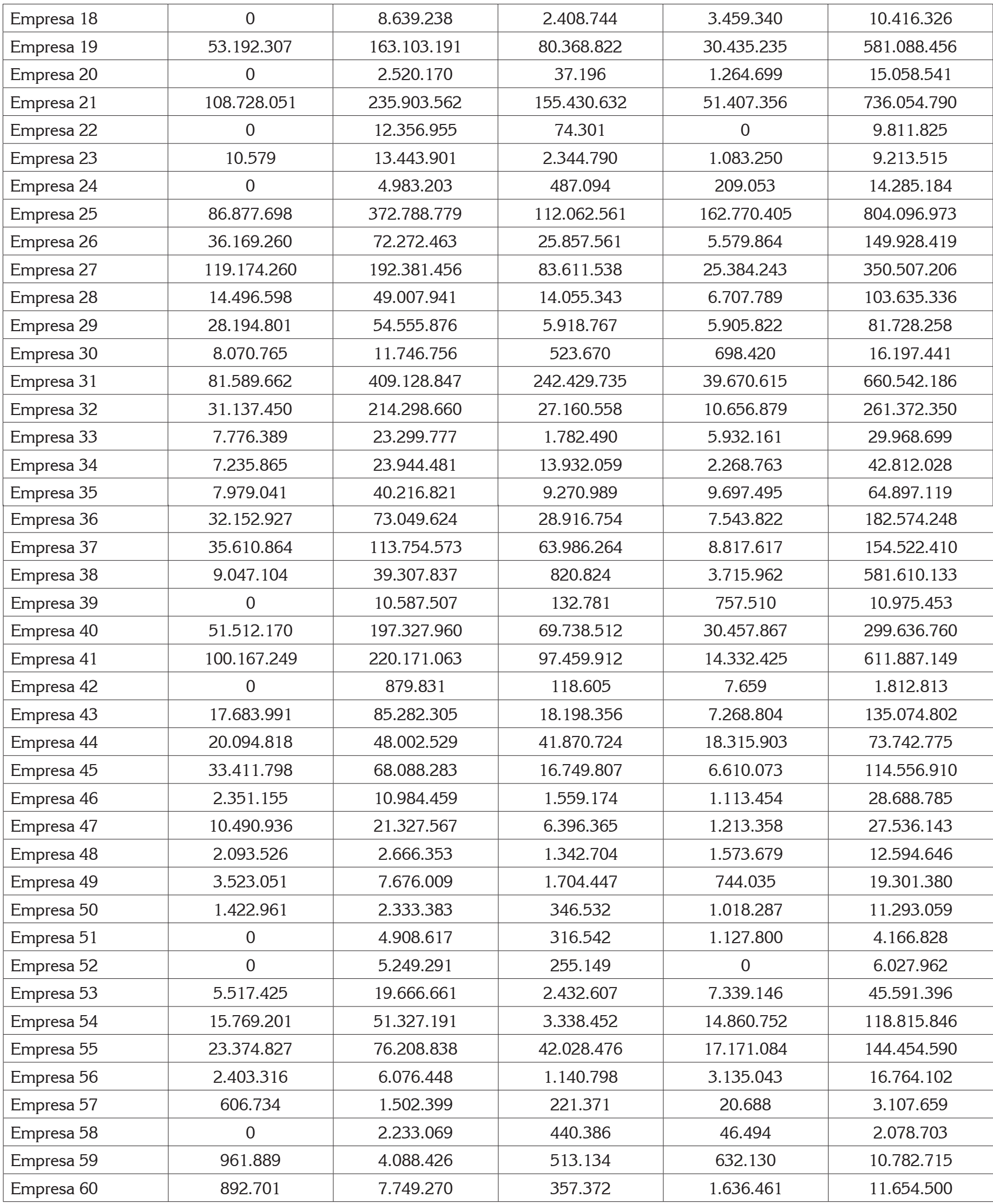

Fuente: Tomada de la Superintendencia de Sociedades, 2010. 
Es importante tener en cuenta que si el número de DMU a evaluar es menor que el número combinado de entradas y de salidas, una gran parte de DMU será consideradas eficientes y el resultado será cuestionable, debido a un número insuficiente de grados de libertad. Una regla básica para que un modelo de Análisis Envolvente de Datos discrimine correctamente entre unidades eficientes y no eficientes es que $n$ (Número de DMU) sea igual o mayor que el máximo entre $\mathrm{m}$ x s o $3 \mathrm{x}(\mathrm{m}+\mathrm{s})$, donde $\mathrm{m}$ es el número de variables de entrada y $\mathrm{s}$ el número de variables de salida (Cooper et al. 2006). Esta regla se cumple en el presente estudio. Para el cálculo de las eficiencias con el modelo CCR-O, se utilizó el software DEA Solver PRO 09, con el cual, se pudo realizar el análisis de los diferentes niveles de eficiencia del grupo de empresas objeto de la investigación.

\section{RESULTADOS Y DISCUSIÓN}

Los resultados de la investigación hacen referencia a: 1) El estudio de la correlación entre las variables de la investigación; 2) Los puntajes de eficiencia de las empresas certificadas con BASC en la ciudad de Medellín; 3) La clasificación de las diferentes organizaciones por tipos de eficiencia y, 4) La proyección de la variable de salida (Ingresos Operacionales) sobre la frontera eficiente. Está muestra el nivel de ingresos operacionales necesario para que una empresa ineficiente logre ser eficiente. Para, finalmente, analizar la relación existente entre la eficiencia organizacional del sector y la estandarización con la norma BASC.

A continuación, se presenta en la tabla 2, la correlación entre las variables del estudio utilizadas para analizar la eficiencia técnica las empresas. Se observa que existe una alta correlación entre las variables internas de las organizaciones con la generación de los ingresos operacionales. Lo anterior es coherente con el hecho que los procesos de estandarización
BASC tienen como intencionalidad generar eficiencia y eficacia, lo cual, requiere una serie de condiciones internas y de disponibilidad de activo corriente, propiedad planta y equipo y recursos disponibles para mejorar los procesos logísticos de las organizaciones donde se implemente.

Luego de evaluar la eficiencia de las 60 empresas certificadas con BASC en Medellín, obtenemos los puntajes de eficiencia CCR -O, que se muestran en la tabla 3. En el modelo CCR, una DMU es eficiente si el puntaje de eficiencia es igual a uno $(\theta=1)$ y no posee holguras (la holgura en todas las variables es igual a cero (0)); en nuestro caso de estudio, las empresas cuyo puntaje de eficiencia es uno (1), no presentan holguras en sus variables, por lo tanto, es suficiente observar que el puntaje de eficiencia sea igual a uno $(\theta=1)$, para identificarla como eficiente. Los resultados indican que 7 de 60 empresas son eficientes, que corresponde al $11,67 \%$ del total de las empresas evaluadas. Debido a que en la evaluación de la eficiencia hemos utilizado un enfoque a salidas, se debe prestar especial interés al inverso del score de eficiencia (1/ Score), presentado en la tabla 3 , para las empresas que presentan ineficiencia, es decir, con $1 /$ Score $>1$, lo que indica el factor, por el cual, se debe multiplicar las variables de salida para alcanzar la eficiencia, de esta manera, para que la Empresa 1 alcance la eficiencia debe multiplicar la magnitud de sus ingresos operacionales 8,692 veces; el resultado se conoce como la proyección en la frontera eficiente. El análisis de la tabla 3 permite aseverar que del grupo de empresas consideradas en este estudio, la empresa 10 presenta el peor desempeño, ya que para alcanzar la eficiencia debe multiplicar sus ingresos operacionales 17,73 veces (incremento en 16,73 veces), seguida de la empresa 33 , que requiere un incremento de 10,5 veces en sus ingresos operacionales y por la empresa 29 , que debe aumentar 9,877 veces sus ingresos operacionales.

Tabla 2. Correlación entre las variables.

\begin{tabular}{|l|c|c|c|c|c|}
\hline & $\begin{array}{c}\text { Subtotal } \\
\text { Inventarios }\end{array}$ & $\begin{array}{c}\text { Total Activo } \\
\text { Corriente }\end{array}$ & $\begin{array}{c}\text { Propiedades } \\
\text { Planta y Equipo }\end{array}$ & Proveedores & $\begin{array}{c}\text { Ingresos } \\
\text { Operacionales }\end{array}$ \\
\hline Subtotal Inventarios & 1 & 1 & & & \\
\hline Total Activo Corriente & 0,88 & 0,91 & 1 & & \\
\hline $\begin{array}{l}\text { Propiedades Planta y } \\
\text { Equipo }\end{array}$ & 0,84 & 0,78 & 0,62 & 1 & \\
\hline Proveedores & 0,67 & 0,89 & 0,83 & 0,74 & 1 \\
\hline $\begin{array}{l}\text { Ingresos Operacion- } \\
\text { ales }\end{array}$ & 0,85 & & & \\
\hline
\end{tabular}

Fuente: Calculado por los autores. 
Tabla 3. Puntajes de eficiencia modelo CCR - O.

\begin{tabular}{|c|c|c|c|c|c|c|c|}
\hline No. & DMU & Score & $1 /$ Score & No. & DMU & Score & $1 /$ Score \\
\hline 1 & Empresa 1 & 0,115 & 8,692 & 31 & Empresa 31 & 0,118 & 8,448 \\
\hline 2 & Empresa 2 & 0,174 & 5,742 & 32 & Empresa 32 & 0,144 & 6,931 \\
\hline 3 & Empresa 3 & 0,135 & 7,420 & 33 & Empresa 33 & 0,087 & 11,504 \\
\hline 4 & Empresa 4 & 0,647 & 1,546 & 34 & Empresa 34 & 0,121 & 8,275 \\
\hline 5 & Empresa 5 & 0,271 & 3,692 & 35 & Empresa 35 & 0,119 & 8,420 \\
\hline 6 & Empresa 6 & 0,266 & 3,755 & 36 & Empresa 36 & 0,169 & 5,920 \\
\hline 7 & Empresa 7 & 0,457 & 2,191 & 37 & Empresa 37 & 0,104 & 9,621 \\
\hline 8 & Empresa 8 & 0,181 & 5,520 & 38 & Empresa 38 & 1,000 & 1,000 \\
\hline 9 & Empresa 9 & 1,000 & 1,000 & 39 & Empresa 39 & 0,637 & 1,570 \\
\hline 10 & Empresa 10 & 0,056 & 17,728 & 40 & Empresa 40 & 0,103 & 9,744 \\
\hline 11 & Empresa 11 & 0,292 & 3,419 & 41 & Empresa 41 & 0,250 & 3,997 \\
\hline 12 & Empresa 12 & 0,472 & 2,117 & 42 & Empresa 42 & 1,000 & 1,000 \\
\hline 13 & Empresa 13 & 0,225 & 4,437 & 43 & Empresa 43 & 0,116 & 8,655 \\
\hline 14 & Empresa 14 & 0,168 & 5,965 & 44 & Empresa 44 & 0,104 & 9,632 \\
\hline 15 & Empresa 15 & 0,931 & 1,074 & 45 & Empresa 45 & 0,114 & 8,794 \\
\hline 16 & Empresa 16 & 0,229 & 4,372 & 46 & Empresa 46 & 0,185 & 5,417 \\
\hline 17 & Empresa 17 & 0,296 & 3,382 & 47 & Empresa 47 & 0,133 & 7,533 \\
\hline 18 & Empresa 18 & 0,218 & 4,588 & 48 & Empresa 48 & 0,319 & 3,132 \\
\hline 19 & Empresa 19 & 0,241 & 4,153 & 49 & Empresa 49 & 0,170 & 5,884 \\
\hline 20 & Empresa 20 & 1,000 & 1,000 & 50 & Empresa 50 & 0,327 & 3,057 \\
\hline 21 & Empresa 21 & 0,211 & 4,742 & 51 & Empresa 51 & 0,205 & 4,873 \\
\hline 22 & Empresa 22 & 1,000 & 1,000 & 52 & Empresa 52 & 1,000 & 1,000 \\
\hline 23 & Empresa 23 & 0,211 & 4,742 & 53 & Empresa 53 & 0,157 & 6,383 \\
\hline 24 & Empresa 24 & 1,000 & 1,000 & 54 & Empresa 54 & 0,156 & 6,392 \\
\hline 25 & Empresa 25 & 0,146 & 6,860 & 55 & Empresa 55 & 0,128 & 7,806 \\
\hline 26 & Empresa 26 & 0,163 & 6,144 & 56 & Empresa 56 & 0,186 & 5,363 \\
\hline 27 & Empresa 27 & 0,123 & 8,121 & 57 & Empresa 57 & 0,631 & 1,584 \\
\hline 28 & Empresa 28 & 0,143 & 6,997 & 58 & Empresa 58 & 0,381 & 2,627 \\
\hline 29 & Empresa 29 & 0,101 & 9,877 & 59 & Empresa 59 & 0,178 & 5,610 \\
\hline 30 & Empresa 30 & 0,141 & 7,071 & 60 & Empresa 60 & 0,153 & 6,527 \\
\hline
\end{tabular}

Fuente: Elaboración de los autores con Información de la Superintendencia de Sociedades, 2010.

A los resultados de eficiencia del modelo utilizado, se les realizó una clasificación en:

- Empresas eficientes $(\theta=1)$

- Empresas con eficiencia alta $(0,80<=\theta<1)$

- Empresas con eficiencia media $(0,60<=\theta<0,80)$

- Empresas con eficiencia baja $(\theta<0,60)$
La tabla 4 muestra la clasificación de las 60 empresas consideradas en el estudio, según su grado de eficiencia. También muestra que sólo el $11,67 \%$ de las empresas consideradas en el estudio son eficientes, es decir, dado los recursos que poseen obtienen los mejores resultados en su ingreso operacional; el 1,67\% de las empresas presenta un desempeño alto; $5 \%$ una eficiencia media y alrededor del $82 \%$ de las empresas con Certificación BASC en la ciudad de Medellín, presentan un índice de eficiencia bajo. 
Tabla 4. Clasificación de las Empresas según su grado de eficiencia.

\begin{tabular}{|c|c|c|c|c|c|}
\hline $\begin{array}{c}\text { Empresas } \\
\text { eficientes }\end{array}$ & $\begin{array}{c}\text { Empresas con } \\
\text { eficiencia alta }\end{array}$ & $\begin{array}{c}\text { Empresas con } \\
\text { eficiencia media }\end{array}$ & \multicolumn{3}{|c|}{ Empresas con eficiencia baja } \\
\hline Empresa 9 & Empresa 15 & Empresa 4 & Empresa 1 & Empresa 23 & Empresa 43 \\
\hline Empresa 20 & & Empresa 39 & Empresa 2 & Empresa 25 & Empresa 44 \\
\hline Empresa 22 & & Empresa 57 & Empresa 3 & Empresa 26 & Empresa 45 \\
\hline Empresa 24 & & Empresa 5 & Empresa 27 & Empresa 46 \\
\hline Empresa 38 & & Empresa 6 & Empresa 28 & Empresa 47 \\
\hline Empresa 42 & & Empresa 7 & Empresa 29 & Empresa 48 \\
\hline Empresa 52 & & Empresa 8 & Empresa 30 & Empresa 49 \\
\hline & & Empresa 10 & Empresa 31 & Empresa 50 \\
\hline & & Empresa 11 & Empresa 32 & Empresa 51 \\
\hline & & Empresa 12 & Empresa 33 & Empresa 53 \\
\hline & & Empresa 13 & Empresa 34 & Empresa 54 \\
\hline & & & Empresa 14 & Empresa 35 & Empresa 55 \\
\hline & & & Empresa 16 & Empresa 36 & Empresa 56 \\
\hline & & & Empresa 18 & Empresa 40 & Empresa 59 \\
\hline & & & Empresa 19 & Empresa 41 & Empresa 60 \\
\hline
\end{tabular}

Fuente: Elaboración de los autores.

También, se realizó un análisis del conjunto de referencia (Figura 1), que muestra las empresas eficientes y el número de empresas ineficientes a las que sirve como par evaluador, convirtiéndose, de esta manera, en referente y, por ende, en candidata para realizar un estudio de benchmarking. Se observa que la empresa 38 aparece 47 veces como par evaluador de otras organizaciones, por lo que se constituye en referente del $88,68 \%$ de las empresas ineficientes y en una unidad productiva que posee un desempeño a imitar. Le siguen las empresas 9 y 20, que son referentes de 26 y 13 unidades organizaciones ineficientes, respectivamente.

Para cada empresa ineficiente, DEA sugiere la combinación de entradas y de salidas necesarias para alcanzar la eficiencia (proyecciones de la DMU ineficiente sobre la frontera eficiente); en el caso de las variables de salida, para que una DMU sea eficiente debe mejorar (aumentar) la magnitud de éstas hasta alcanzar dicha proyección.

La tabla 5 muestra la proyección de la variable de salida para cada empresa ineficiente y la magnitud del ingreso operacional requerido para que una DMU ineficiente alcance la efi- ciencia. Se considera únicamente la variable de salida, dado que se utilizó el modelo CCR-O y propende por determinar cuáles serían las salidas para que una DMU sea eficiente. Al multiplicar los ingresos operacionales de la tabla 1 con el inverso del score (1/Score) de la tabla 3 , se obtiene su proyección sobre la frontera eficiente, mostrada en la tabla 5.

Al analizar los resultados, se puede aseverar que la eficiencia promedio de las organizaciones certificadas en BASC Medellín-Colombia es del 31,85\%, el cual, es bastante bajo y solamente 9 de las 53 unidades ineficientes $(16,98 \%)$ presentan eficiencia relativa por encima del promedio; por otro lado, la mediana de la eficiencia está en $18,30 \%$, un valor mucho menor que la media, indicando que $50 \%$ de las empresas estudiadas presentan eficiencia menor o igual a este valor y que la distribución de la eficiencia indica un sesgo positivo; en este sentido, se puede considerar que las empresas eficientes se comportan como atípicas, dada la distribución de la eficiencia.

Si bien un grupo de siete empresas certificadas en BASC presentó eficiencia, el grupo en general no mostró los mis- 


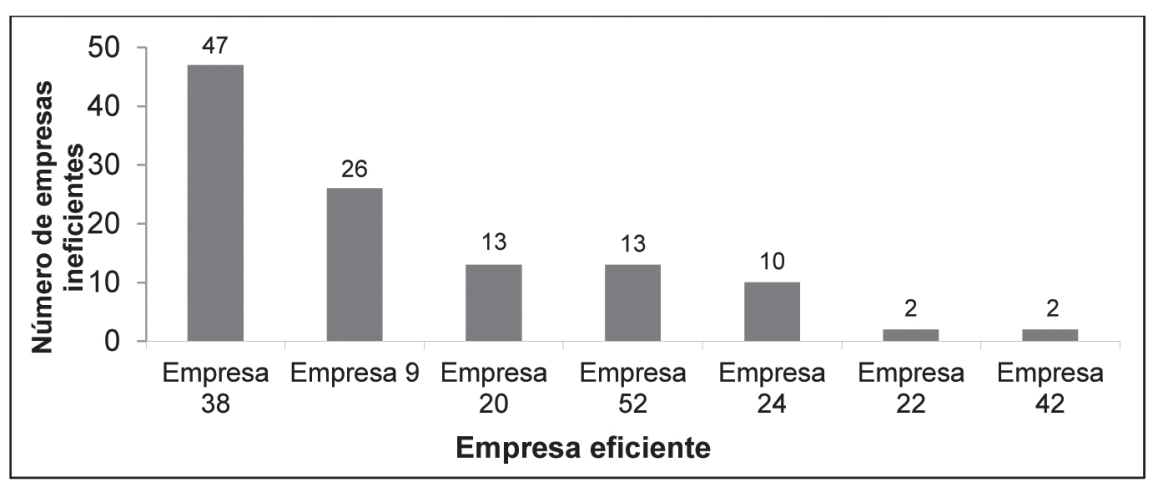

Figura 1. Número de veces que una empresa eficiente es par evaluador de las ineficientes.

Tabla 5. Proyección de los ingresos operacionales sobre la frontera eficiente para que una empresa ineficiente alcance la eficiencia.

\begin{tabular}{|c|c|c|c|c|c|c|c|}
\hline No. & Empresa & Eficiencia & $\begin{array}{l}\text { Proyección en los } \\
\text { Ingresos Operacionales }\end{array}$ & No. & Empresa & Eficiencia & $\begin{array}{l}\text { Proyección en los } \\
\text { Ingresos Operacionales }\end{array}$ \\
\hline 1 & Empresa 1 & 0,115 & 2.970 .259 .015 & 32 & Empresa 32 & 0,144 & 1.811 .623 .692 \\
\hline 2 & Empresa 2 & 0,174 & 104.889 .694 & 33 & Empresa 33 & 0,087 & 344.750 .244 \\
\hline 3 & Empresa 3 & 0,135 & 345.636 .462 & 34 & Empresa 34 & 0,121 & 354.289 .471 \\
\hline 4 & Empresa 4 & 0,647 & 141.967 .839 & 35 & Empresa 35 & 0,119 & 546.457 .194 \\
\hline 5 & Empresa 5 & 0,271 & 438.221 & 36 & Empresa 36 & 0,169 & 1.080 .863 .379 \\
\hline 6 & Empresa 6 & 0,266 & 332.075 .935 & 37 & Empresa 37 & 0,104 & 1.486 .598 .016 \\
\hline 7 & Empresa 7 & 0,457 & 23.244 .027 & 39 & Empresa 39 & 0,637 & 17.235 .165 \\
\hline 8 & Empresa 8 & 0,181 & 217.962 .497 & 40 & Empresa 40 & 0,103 & 2.919 .721 .608 \\
\hline 10 & Empresa 10 & 0,056 & 437.458 .186 & 41 & Empresa 41 & 0,250 & 2.445 .637 .633 \\
\hline 11 & Empresa 11 & 0,292 & 25.810 .157 & 43 & Empresa 43 & 0,116 & 1.169 .038 .936 \\
\hline 12 & Empresa 12 & 0,472 & 6.420 .160 & 44 & Empresa 44 & 0,104 & 710.259 .312 \\
\hline 13 & Empresa 13 & 0,225 & 542.300 .298 & 45 & Empresa 45 & 0,114 & 1.007 .453 .942 \\
\hline 14 & Empresa 14 & 0,168 & 128.891 .809 & 46 & Empresa 46 & 0,185 & 155.403 .373 \\
\hline 15 & Empresa 15 & 0,931 & 2.134 .286 & 47 & Empresa 47 & 0,133 & 207.432 .150 \\
\hline 16 & Empresa 16 & 0,229 & 21.747 .295 & 48 & Empresa 48 & 0,319 & 39.452 .131 \\
\hline 17 & Empresa 17 & 0,296 & 54.151 .993 & 49 & Empresa 49 & 0,170 & 113.576 .451 \\
\hline 18 & Empresa 18 & 0,218 & 47.786 .057 & 50 & Empresa 50 & 0,327 & 34.525 .410 \\
\hline 19 & Empresa 19 & 0,241 & 2.413.322.020 & 51 & Empresa 51 & 0,205 & 20.305 .809 \\
\hline 21 & Empresa 21 & 0,211 & 3.490 .497 .380 & 53 & Empresa 53 & 0,157 & 290.993 .608 \\
\hline 23 & Empresa 23 & 0,211 & 43.689 .254 & 54 & Empresa 54 & 0,156 & 759.451 .974 \\
\hline 25 & Empresa 25 & 0,146 & 5.515 .890 .669 & 55 & Empresa 55 & 0,128 & 1.127 .608 .024 \\
\hline 26 & Empresa 26 & 0,163 & 921.152 .238 & 56 & Empresa 56 & 0,186 & 89.908 .883 \\
\hline 27 & Empresa 27 & 0,123 & 2.846 .531 .703 & 57 & Empresa 57 & 0,631 & 4.921 .867 \\
\hline 28 & Empresa 28 & 0,143 & 725.135 .679 & 58 & Empresa 58 & 0,381 & 5.460 .452 \\
\hline 29 & Empresa 29 & 0,101 & 807.224 .531 & 59 & Empresa 59 & 0,178 & 60.493 .534 \\
\hline 30 & Empresa 30 & 0,141 & 114.533 .898 & 60 & Empresa 60 & 0,153 & 76.066 .624 \\
\hline 31 & Empresa 31 & 0,118 & 5.579 .974 .665 & & & & \\
\hline
\end{tabular}

Fuente: Elaboración de los autores con información de la Superintendencia. 
mos resultados, por lo que podemos afirmar que la certificación BASC no afecta de forma significativa el incremento en la eficiencia, cuando se analiza con las variables de entrada consideradas para esta investigación, como son: el total de inventarios, los activos corriente, propiedad planta y equipo y el rubro proveedores.

Conflicto de intereses: El manuscrito fue preparado y revisado con la participación de todos los autores, quienes declaramos que no existe ningún conflicto de intereses, que ponga en riesgo la validez de los resultados presentados.

\section{BIBLIOGRAFÍA}

1. BANKER, R.; CHARNES, A.; COOPER, W. 1984. Some models for estimating technical and scale inefficiencies in data envelopment analysis. Manag. Sci. 30(9):1078-1092.

2. BELMONTE, L.; PLAZA, J. 2008. Análisis de la eficiencia en las cooperativas de crédito en España. Una propuesta metodológica basada en el análisis envolvente de datos (DEA). Ciriec-España. 63:113-133.

3. CHARNES, A.; COOPER, W.; RHODES, E. 1978. Measuring the efficiency of decision making units. Eur. J. Operat. Res. 2(6):429-444.

4. COLL, V.; BLASCO, O. 2007. Evaluación de la Eficiencia de la Industria Textil Española a Partir de Información Económico-Financiera: Una Aplicación del Análisis Envolvente de Datos. Rev. Invest. Opera. 28(1):6191.

5. COOPER, W.; SEIFORD, L.; TONE, K. 2006. Introduction to Data Envelopment Analysis and Its Uses. Springer Science Business Media, Inc. (New York, NY). p.105-106.

6. GARCÍA, J.; GONZÁLEZ, E. 2005 .Aplicación y Utilidad del Análisis Envolvente de Datos en la medida de la eficiencia de los equipos de atención primaria de Austrias. Disponible desde Internet en: http://www. fgcasal.org/aes/docs/VicenteGarciaComunicacionJornadasAES1.pdf (con acceso el 05/03/13).

7. HADI-VENCHEH, A.; ASGHAR, A.; SOLEIMANI-DAMANEH, M. 2008. A DEA model for resource allocation. Econ. Modelling. 25(5):983-993.

8. HERNÁNDEZ, M. 2004. Importancia del BASC en el Comercio Exterior. Memorias Asamblea Asociación Latinoamericana de Logística. Montevideo, Uruguay. p.2-6.
9. MARTÍN, N.; HERNAGÓMEZ, J.; MARTÍN, V. 2007. El deleite de la eficiencia. Disponible desde Internet en: http://ubr.universia.net/pdfs/ubr0022007056.pdf (con acceso el 04/18/13).

10. MEJÍA, G.; CASTRO, E. 2007. Optimización del proceso logístico en una empresa colombiana de alimentos congelados y refrigerantes. Rev.Ing. 26:47-54.

11. NIJKAMP, P.; SUZUKI, P. 2009. A Generalized Goalsachievement Model in Data Envelopment Analysis: an Application to Efficiency Improvement in Local Government Finance in Japan. Spatial Econ. Anal. 4(3):249-274.

12. PARADI, J.; ASMILD, M.; SIMAK, P. 2004. Using DEA and Worst Practice DEA in Credit Risk Evaluation. J. Product. Anal. 21(2):153-165.

13. PASIOURAS, F. 2008. Estimating the technical and scale efficiency of Greek commercial banks: The impact of credit risk, off-balance sheet activities, and international operations. Res. Int. Business Finance. 22(1):301-308.

14. PASTOR, J.; LOVELL, C; TULKENS, H. 2006. Evaluating the financial performance of bank branches. Ann. Operat. Res. 145(1):321-337.

15. PERDOMO, A; MENDIETA, J. 2007. Factores que afectan la eficiencia técnica y asignativa en el sector cafetero colombiano: Una aplicación con análisis envolvente de datos. Desarrollo y Sociedad. 10(2):1-45.

16. PINO, J.; SOLÍS, F.; DELGADO, M.; BAREA, R. 2010. Evaluación de la eficiencia de grupos de investigación mediante el Análisis Envolvente de Datos (DEA). Prof. Inform. 19(2):160-167.

17. PRIMORAC, M.; TROSKOT, Z. 2005. Measuring the efficiency and productivity of the Croatian banks with Malmquist's index of change of total factor productivity. Financial Theory and Practice. 29(4):309-325.

18. PSILLAKI, M.; TSOLAS, I.; MARGARITI, D. 2010. Evaluation of credit risk based on firm performance. Eur. J. Operat. Res. 201(3):873-881.

19. QUINDOS, M.; RUBIERA, F.; VICENTE, M. 2003. Análisis de la eficiencia en el sector de los servicios avanzados a las empresas: una aplicación para el caso del principado de Asturias, Depto. De Economía Aplicada, Universidad de Oviedo. Disponible desde in- 
ternet en: http://www.uv.es/asepuma/XI/21.pdf (con acceso el 12/08/2013).

20. QUINTANILHA, J.; CORREIA, J. 2012. Evaluación de la eficiencia de las compañías aéreas brasileñas a través de un modelo híbrido de análisis envolvente de datos (DEA) y programación lineal multi objetivo. Ingeniare. Rev. chilena Ing. 20(3):331-342.

21. TOLOO, M.; NALCHIGAR, S. 2009. A new integrated DEA model for finding most BCC-efficient DMU. Appl. Math. Mod. 33(1):597-604.
22. TORTOSA-AUSINA, E.; GRIFELL-TATJE, E.; ARMERO, C.; CONESA, D. 2008. Sensitivity analysis of efficiency and Malmquist productivity indices: An application to Spanish savings banks. Eur. J. Opera. Res. 184(3):1062-1084.

23. WONG, W.; WONG, K. 2007. Supply chain performance measurement system using DEA modeling. Indust. Manag. Data Syst. 107(3):361-381.

Recibido: Junio 19 de 2013

Aceptado: Noviembre 15 de 2013

\section{Como citar:}

Fontalvo Herrera, T.J.; Mendoza Mendoza, A.; Visbal Cadavid, D. 2014. Eficiencia en los procesos logísticos en las empresas certificadas en BASC Medellin mediante análisis envolvente de datos. Rev. U.D.C.A Act. \& Div. Cient. 17(1): 265-274. 